\title{
Null Vectors, Spinors, and Strings
}

\author{
P. Budinich \\ International School for Advanced Studies, I-34014 Trieste, Italy
}

\begin{abstract}
It is shown how, in the frame of the Cartan-conception of spinors, the old theorems on minimal surfaces, as generated from null-curves, formulated by Enneper-Weierstrass (1864-1866) for 3-dimensional ordinary space, and by Eisenhart (1911) for 4-dimensional space-time, may be reformulated in terms of complex 2- and 4-component projective spinors respectively. For the corresponding real (Majorana) spinors instead the same procedure naturally leads to strings in 3-dimensional and 4-dimensional space-time $\left(\mathbb{R}^{2,1}\right.$ and $\left.\mathbb{R}^{3,1}\right)$. It is suggested that this close connection with Cartan-spinors, and the corresponding (projective) null-geometry, may be the clue for understanding the fundamental nature of strings.
\end{abstract}

\section{Introduction}

For more than a century [1], mathematicians have known the fundamental, elementary character of null vectors and null lines and, in particular, of their property to generate minimal surfaces. These played subsequently a central role in several later developments of mathematics, geometry and, especially, of those fundamental branches of physics which are based on classical and quantum field theories.

E. Cartan discovered spinors in 1913 [2] in searching for new representations of rotation groups. However, from his subsequent work [3], it clearly appears how he was especially struck by the equivalence of what he named "simple spinors" with null or isotropic vectors and totally null planes. A two-component spinor, in his words: "est donc en quelque sort un vecteur isotrope orienté ou polarisé" and, in general: "tout spineur simple peut être défini d'une manière concrete comme un $v$-vecteur isotrope polarisé." This equivalence may now be expressed in modern, perhaps more rigorous, language as a bijective map: "the Cartan-map" [4] between simple or pure-spinor-directions and geometrical elements (totally null planes, quadric Grassmanians or flags) in projective spaces. This Cartan-map is, in 
our opinion, the optimal starting point for understanding the basic role of nullvectors and of spinors ${ }^{1}$ in geometry, and then in physics [4, 5].

The concept, at first sight surprising and peculiar, of relativistic strings was introduced in physics, almost incidentally in 1969 [8]. Only recently [9] this concept has manifested its virtue as a possible effective tool for overcoming at least some of the severe difficulties which have plagued relativistic quantum field theories for more than forty years. Should these properties be confirmed, as more and more signs seem to indicate, then it would not be surprising if strings were related to fundamental geometrical objects like null vectors and pure spinors, and this would, in some way, allow a better understanding of their properties and to grasp the deep origin of their virtues.

I wish to show in this paper that, if one adopts the Cartan conception of spinors, then the old general results regarding minimal surfaces in ordinary 3-dimensional space and 4-dimensional space-time may be reformulated in terms of complex spinor-directions [see Eqs. (4), (4'), (5), and (17), (18), (18'), (19)]; the corresponding equations with real spinors instead represent strings, obviously in spaces which admit those real spinors, that is 3-and 4-dimensional space-time [see Eqs. (12), (12) for $\mathbb{R}^{2,1}$ and (25) for $\mathbb{R}^{3,1}$.

\section{Null Curves in $\mathbb{C}^{3}$. Minimal Surfaces in $\mathbb{R}^{3}$ and Strings in $\mathbb{R}^{2,1}$ as Generated from Two-Component Complex and Real Spinors Respectively}

Let $\varphi\rangle:=\left|\begin{array}{l}\varphi_{0} \\ \varphi_{1}\end{array}\right|$ represent a $\mathbb{C}^{3}$-spinor thought of as a complex vector in a two dimensional spinor-space $S=\mathbb{C}^{2}$. According to E. Cartan there is a two to one correspondence between $\varphi$ and a null vector $\bar{Z}:\left(Z_{1}^{2}+Z_{2}^{2}+Z_{3}^{2}=0\right)$ of $\mathbb{C}^{3}$. This is a particular case of the Cartan-map $S \rightarrow \mathbb{C}^{N}$ defined in a previous paper [4] and bijectively connecting pure spinor directions with elements of a projective quadric: in this case $Q_{1}:\left\{Z^{2}=0, Z \in \mathbb{C}^{3}\right\}$.

Defining $\langle\varphi=:-\varphi\rangle^{T} i \sigma_{2}$, the Cartan-map may be represented by the matrix equation:

$$
\varphi\rangle\left\langle\varphi=Z_{j} \sigma^{j} ;\left|\begin{array}{cc}
\varphi_{0} \varphi_{1} & -\varphi_{0}^{2} \\
\varphi_{1}^{2} & -\varphi_{0} \varphi_{1}
\end{array}\right|=\left|\begin{array}{cc}
Z_{3} & Z_{1}-i Z_{2} \\
Z_{1}+i Z_{2} & -Z_{3}
\end{array}\right|\right.
$$

(we may multiply both members by an arbitrary non-null complex factor), where $Z_{j}$ are (orthonormal) coordinates (projective) of $Q_{1}, \varphi_{A}$ of $\varphi$ (projective), $\sigma^{j}$ are Pauli matrices generating $\mathbb{C}^{3}$ Clifford algebra: $\mathrm{Cl}\left(\mathbb{C}^{3}\right)$.

$$
\begin{aligned}
& \text { From (1) we get, for any } \left.{ }^{2} \varphi\right\rangle=\left|\begin{array}{c}
\varphi_{0} \\
\varphi_{1}
\end{array}\right|: Z_{j}=\frac{1}{2}\left\langle\varphi \sigma_{j} \varphi\right\rangle \in Q_{1} \text {, explicitly: } \\
& \qquad Z_{1}=\frac{1}{2}\left(\varphi_{1}^{2}-\varphi_{0}^{2}\right), \quad Z_{2}=\frac{-i}{2}\left(\varphi_{0}^{2}+\varphi_{1}^{2}\right), \quad Z_{3}=\varphi_{0} \varphi_{1}
\end{aligned}
$$

\footnotetext{
${ }^{1}$ After the advent of quantum mechanics, the discoveries of the Dirac equation and of relativistic field theories, several physicists, on various occasions, have conjectured the fundamental role of spinors in the explanation of elementary physical phenomena [6]. However, the Cartan conception of pure spinors, that is their strict connection with the elementary geometry of null vectors and totally null planes, was only rarely [7] considered as relevant for physics

${ }^{2}$ If we substitute $\varphi_{0}, \varphi_{1}$ with holomorphic functions of a complex variable $\zeta$, then $Z_{j}(\zeta)$ may be considered as components of the tangent to a null line in $\mathbb{C}^{3}$
} 
We may represent locally the direction $\varphi_{t}$ of $\varphi$ by the complex number $\zeta=\varphi_{0} / \varphi_{1}$, for $\left.\varphi_{1} \neq 0: \varphi_{t}\right\rangle=\left|\begin{array}{l}\zeta \\ 1\end{array}\right|$, and indicating with $t_{j}$ the corresponding chart for $Z_{j}$-direction as implied by Eq. (1):

$$
\begin{aligned}
& Z_{1} \rightarrow t_{1}(\zeta)=\frac{1}{2}\left\langle\varphi_{t} \sigma_{1} \cdot \varphi_{t}\right\rangle=\frac{1}{2}\left(1-\zeta^{2}\right), \\
& Z_{2} \rightarrow t_{2}(\zeta)=\frac{1}{2}\left\langle\varphi_{t} \sigma_{2} \cdot \varphi_{t}\right\rangle=-\frac{i}{2}\left(1+\zeta^{2}\right), \\
& Z_{3} \rightarrow t_{3}(\zeta)=\frac{1}{2}\left\langle\varphi_{t} \sigma_{3} \cdot \varphi_{t}\right\rangle=\zeta .
\end{aligned}
$$

Consider the products: $t_{j}(\zeta) F(\zeta)$, with $F(\zeta)$ an arbitrary holomorphic function of $\zeta$, they may be considered as the null tangents at $\zeta$ of a class of holomorphic null lines in $\mathbb{C}^{3}$. Any one line of such a class will then be represented by its orthonormal coordinates $T_{j}(\zeta)$ :

$$
T_{j}(\zeta)=T_{j}(0)+\int_{0}^{\zeta} \frac{1}{2}\left\langle\varphi_{t} \sigma_{j} \varphi_{t}\right\rangle F(\zeta) d \zeta,
$$

where the integral is performed along any path in the complex plane $\zeta$. Setting $T_{j}=X_{j}+i Y_{j}$ and $\zeta=u+i v$, we immediately obtain the Enneper-Weierstrass [10] parametric representation ${ }^{3}$ of any minimal surface $X_{j}(u, v)$ in $\mathbb{R}^{3}$ [insert in (3) expressions (2)]:

$$
X_{j}(u, v)=X_{j}(0,0)+\operatorname{Re} \int_{0}^{\zeta} \frac{1}{2}\left\langle\varphi_{t} \sigma_{j} \varphi_{t}\right\rangle F(\zeta) d \zeta .
$$

Setting $F(\zeta)=f^{\prime \prime \prime}(\zeta)$ we get the differential form of it as given by Hitchin [11] (with appropriate values of $X_{j}$ and $f$ at $\zeta=0$ ).

$$
\begin{aligned}
& X_{1}(u, v)=\operatorname{Re}\left[\frac{1}{2}\left(1-\zeta^{2}\right) f^{\prime \prime}+\zeta f^{\prime}-f\right], \\
& X_{2}(u, v)=\operatorname{Re}\left[\frac{-i}{2}\left(1+\zeta^{2}\right) f^{\prime \prime}+i \zeta f^{\prime}-i f\right], \\
& X_{3}(u, v)=\operatorname{Re}\left[\zeta f^{\prime \prime}-f^{\prime}\right]
\end{aligned}
$$

(connected to the spectral curve of a static monopole in $\mathbb{R}^{3}$ ).

The appearence in (3) and (4) of an arbitrary holomorphic function $F(\zeta)$ may suggest the possibility of deriving the above formalism from Weyl space-time spinors. In fact the complex variable $\zeta$ may be thought of as characterizing the (local) stereographic projection of the $\mathbb{R}^{3,1}$ celestial Riemann sphere $S_{2}:\left\{\chi_{\mu} \chi^{\mu}=0\right.$, $\left.t=1 ; \chi_{\mu} \in \mathbb{R}^{3,1}\right\}$ on an equatorial plane. Then $\zeta=\varphi_{0} / \varphi_{1}$, where $\varphi=\left|\begin{array}{l}\varphi_{0} \\ \varphi_{1}\end{array}\right|$ represents a Weyl, space-time spinor ${ }^{4}$. Equivalently this result may also be obtained algebraically by rewriting the Cartan-map-Eq. (1) in the form:

$$
\varphi\rangle\left\langle\varphi=Z^{j} \sigma_{j}=\left(E^{j}+i H^{j}\right) \sigma_{j}=\frac{1}{4} f^{\mu \nu}\left[\gamma_{\mu}, \gamma_{v}\right]\left(1+\gamma_{5}\right),\right.
$$

\footnotetext{
${ }^{3}$ In the hypothesis of the preceeding footnote $F(\zeta)$ may be eliminated in Eqs. (3) and (4) and the form (94) of Weierstrass formula of [10] is obtained

${ }^{4}$ This allows a simple geometrical interpretation of the arbitrary holomorphic function $F(\zeta)$. In fact we could equivalently take, instead of the plane section $t=1$ of the light cone, giving $S_{2}$, any other one conformally mapped to the Riemann sphere $S_{2}$, this arbitrary change of section would produce the appearance of the arbitrary function $F(\zeta)$
} 
where $\gamma_{\mu}$ are the generators of $\mathbb{R}^{3,1}$-Clifford algebra $\mathrm{Cl}(3,1)\left(\gamma_{5}^{2}=1\right) ; f^{\mu \nu}$ behaves like the antiself-dual e.m. tensor and use was made of the algebraic identity $\sigma_{1} \sigma_{2} \sigma_{3}$ $\equiv i \mathbb{1}$. The $Z$ isotropy is now expressed by: $\bar{E}^{2}=\bar{H}^{2} ; \bar{E} \cdot \bar{H}=0$.

Also the chosen local chart of $\varphi=\left|\begin{array}{l}\varphi_{0} \\ \varphi_{1}\end{array}\right| \rightarrow \varphi_{t}=\left|\begin{array}{c}\zeta \\ 1\end{array}\right|$ corresponds to:

$$
Z_{1}^{2}+Z_{2}^{2}+Z_{3}^{2}=0 \rightarrow \frac{Z_{1}-i Z_{2}}{Z_{1}+i Z_{2}}+\frac{Z_{3}^{2}}{\left(Z+i Z_{2}\right)^{2}}=0
$$

and, in the Cartan-map Eq. (1):

$$
\varphi\rangle\left\langle\varphi=Z^{j} \sigma_{j} \rightarrow\left|\begin{array}{cc}
\zeta & -\zeta^{2} \\
\varphi_{1}^{2}=1 & -\zeta
\end{array}\right|=\left|\begin{array}{cc}
t_{3} & -t_{3}^{2} \\
\left(Z_{1}+i Z_{2}\right)=1 & -t_{3}
\end{array}\right| .\right.
$$

In such a way that, in the chosen local chart, the complex variable $\zeta$ has a precise physical ${ }^{5}$ meaning: $\zeta=t_{3}=E_{3}+i H_{3}$ (for $E_{1}=H_{2}+1 ; E_{2}=-H_{1}$ ).

We may now express the minimal surface-coordinates $X_{j}$ in terms of the Weyl spinor $\varphi$ and of the one $\dot{\varphi}$, of opposite chirality, for which: $\dot{\varphi}\rangle\left\langle\dot{\varphi}=\left(E_{j}-i H_{j}\right) \sigma^{j}[\right.$ see Eq. (14) for signs]. Then any $\mathbb{R}^{3}$-minimal surface in Eq. (4) results by summing the contribution from both such Weyl spinor directions; Enneper-Weierstrass Eq. (4) takes the spinorial form:

$$
\chi_{j}(u, v)=\chi_{j}(0,0)+\int_{0}^{\zeta}\left\langle\varphi_{t} \sigma_{j} \varphi_{t}\right\rangle F(\zeta) d \zeta+\int_{0}^{\bar{\zeta}}\left\langle\dot{\varphi}_{t} \sigma_{j} \dot{\varphi}_{t}\right\rangle \bar{F}(\bar{\zeta}) d \bar{\zeta} .
$$

$\mathbb{R}^{3,1}$-Weyl spinors are complex while $\mathbb{R}^{2,2}$-ones may be real; therefore if we apply to them the Cartan-map and the subsequent derivation above, we will obtain the real counterpart of $\mathbb{R}^{3}$-minimal surfaces: that is, strings in 3-dimensional space-time $\left(\mathbb{R}^{2,1}\right)$ as I am going to show now.

Let $\phi^{+}$and $\phi^{-}$represent two pure (Weyl) spinors of $\mathbb{R}^{2,2}$ of opposite chirality. Since $\mathrm{Cl}(2,2)=R(4)$ and $\mathrm{Cl}^{+}(2,2)=R(2) \oplus R(2)$ (we indicate with $\mathrm{Cl}^{+}$the even Clifford-sub-algebra) they both may be real. We may express for each of them the Cartan-map by [see Eq. (14) with $\varphi, \dot{\varphi} \rightarrow \phi_{+}, \phi_{-}$for signs]:

$$
\left.\phi^{ \pm}\right\rangle\left\langle\phi^{ \pm}=\frac{ \pm 1}{2} f^{j k}\left[\varepsilon_{j}, \varepsilon_{k}\right]+\mathscr{E}_{j} \varepsilon^{j}=\left(\mathscr{E}_{j} \pm \mathscr{H}_{j}\right) \varepsilon^{j}=D_{j}^{ \pm} \varepsilon^{j},\right.
$$

where $\varepsilon_{1}=\sigma_{1} ; \varepsilon_{2}=-i \sigma_{2} ; \varepsilon_{3}=\sigma_{3}$ are the generators of $\mathrm{Cl}(2,1)$ and $D_{j}^{ \pm}=\mathscr{E}_{j} \pm \mathscr{H}_{j}$ are the components of two independent real null vectors in $\mathbb{R}^{2,1}$ (light rays in 3-dimensional space-time). In matrix form:

$$
\left.\phi^{ \pm}\right\rangle\left\langle\phi^{ \pm}=\left|\begin{array}{cc}
\phi_{0} \phi_{1} & -\phi_{0}^{2} \\
\phi_{1}^{2} & -\phi_{0} \phi_{1}
\end{array}\right|=\left|\begin{array}{cc}
D_{3} & D_{1}-D_{2} \\
D_{1}+D_{2} & -D_{3}
\end{array}\right|=D^{ \pm j} \varepsilon_{j}\right.
$$

valid independently for both $\phi^{+}$and $\phi^{-}$, and then both $\left(\bar{D}^{+}\right)^{2}=0$ and $\left(\bar{D}^{-}\right)^{2}=0$ which implies

$$
\overline{\mathscr{E}}^{2}+\overline{\mathscr{H}}^{2}=0 \quad \overline{\mathscr{E}} \cdot \overline{\mathscr{H}}=0,
$$

however with the metric $(+-+)$.

\footnotetext{
${ }^{5}$ In the hypothesis of the fundamental role of spinor- or null-vector-geometry one should expect to discover the central role both of the most natural null vectors we see in nature: light rays, and of the null- or optical-geometry based on them [12]
} 
We may again select a particular local chart for representing pure spinor directions $\phi_{t}^{ \pm}$in terms of projective quadric variables ${ }^{6}$ :

$$
\alpha_{ \pm}=\frac{\phi_{0}^{ \pm}}{\phi_{1}^{ \pm}}=\mathscr{E}_{3} \pm \mathscr{H}_{3}=\sigma \pm \tau \quad\left(\text { for } \phi_{1}^{ \pm} \neq 0 \quad \text { and } \quad D_{1}^{ \pm}+D_{2}^{ \pm} \neq 0\right)
$$

(we set $\mathscr{H}_{3}=\tau, \mathscr{E}_{3}=\sigma$ ). In terms of these parameters (9') becomes:

$$
\left|\begin{array}{cc}
\alpha & -\alpha^{2} \\
\phi_{1}^{2}=1 & -\alpha
\end{array}\right|=\left|\begin{array}{cc}
t_{3} & -t_{3}^{2} \\
D_{1}+D_{2}=1 & -t_{3}
\end{array}\right| ; t_{j}=\frac{1}{2}\left\langle\phi_{t}\left|\varepsilon_{j}\right| \phi_{t}\right\rangle ;
$$

for each $\alpha^{+}, \alpha^{-}, t^{+}, t^{-}$; explicitly:

$$
t_{1}^{ \pm}=\frac{1}{2}\left(1-\alpha_{ \pm}^{2}\right) ; \quad t_{2}^{ \pm}=\frac{1}{2}\left(1+\alpha_{ \pm}^{2}\right) ; \quad t_{3}^{ \pm}=\alpha_{ \pm},
$$

and $t^{ \pm}$may be thought of as the null tangents of two optical-curves in $R^{2,1}$ parametrized by $\alpha_{ \pm}=\sigma \pm \tau$. We then easily obtain a general string in $\mathbb{R}^{2,1}$ in the form:

$$
X_{j}(\sigma, \tau)=X_{j}(0,0)+\int_{0}^{\sigma+\tau}\left\langle\phi_{t}^{+} \varepsilon_{j} \phi_{t}^{+}\right\rangle F\left(\alpha^{+}\right) d \alpha^{+}+\int_{0}^{\sigma-\tau}\left\langle\phi_{t}^{-} \varepsilon_{j} \phi_{t}^{-}\right\rangle F\left(\alpha^{-}\right) d \alpha^{-}
$$

or equivalently

$$
X_{j}(\sigma, \tau)=X_{j}\left(\alpha^{+}\right)+X_{j}\left(\alpha^{-}\right),
$$

where $X_{j}$ are obtained from Eq. (5) by substituting the complex variable $\zeta$ with the real one $\alpha^{+}$or $\alpha^{-}$and eliminating the factor $i$ in $X_{2}$ and the symbol Re.

It is easy to see that $\chi_{j}(\sigma, \tau)$ in (12) and $\left(12^{\prime}\right)$ satisfy the string equations in 3-dimensional space-time $\mathbb{R}^{2,1}$ :

$$
\frac{\partial^{2} x_{j}}{\partial \sigma^{2}}-\frac{\partial^{2} x_{j}}{\partial \tau^{2}}=0, \quad j=1,2,3 ; \quad\left(\frac{\partial x_{j}}{\partial \sigma} \pm \frac{\partial x_{j}}{\partial \tau}\right)^{2}=0
$$

\section{Null Curves in $\mathbb{C}^{4}$. Minimal Surfaces in $\mathbb{R}^{3,1}$ and $\mathbb{R}^{4}$ Generated from Complex Spinors. Strings in $\mathbb{R}^{2,2}$ Generated from Real Spinors}

Let us now consider a $\mathbb{R}^{4,1}$ 4-component pure ${ }^{7}$ spinor $\left.\psi\right\rangle:=\left|\begin{array}{l}\varphi \\ \lambda\end{array}\right| \in S=\mathbb{C}^{4}$.

From the Cartan-map matrix equation:

$$
\psi\rangle\left\langle\psi=\left|\begin{array}{ll}
\varphi\rangle\langle\varphi & -\varphi\rangle\langle\chi \\
\chi\rangle\langle\varphi & -\chi\rangle\langle\chi
\end{array}\right|=\frac{1}{2} f^{\mu v}\left[\gamma_{\mu}, \gamma_{v}\right]+Z^{\mu} \gamma_{\mu} \gamma_{5},\right.
$$

where $\left\langle\psi=-\psi^{T} i \gamma_{2} \gamma_{0}\right.$, we get

$$
Z_{\mu}=\frac{1}{4}\left\langle\psi \gamma_{\mu} \gamma_{5} \psi\right\rangle
$$

\footnotetext{
${ }^{6}$ Like the complex variable $\zeta$, also $\alpha^{ \pm}$may be thought of as resulting from a "stereographic" projection: this time of a torus representing the rays of the $R^{2,2}$ projective quadric: $X_{1}^{2}+X_{3}^{2}$ $=X_{2}^{2}+X_{4}^{2}=1$ (actually two independent ones from the two circles)

${ }^{7} \psi$-pure implies the Cartan-map between projective $\psi$ in $S$ and projective elements of $\mathbb{C}^{5}$ or $\mathbb{C}^{6}$ (twistors). Only for 8- or higher-component spinors constraint equations on spinor components will appear
} 
and for every $\psi, Z$ is null ${ }^{8}$ in $R^{3,1}: Z_{\mu} Z^{\mu}=0$. We recall that the Cartan-definition of $\psi$ as a pure $R^{4,1}$ spinor implies the rank 3 of eq.

$$
\sum_{a=1}^{5} p_{a} \gamma^{a} \psi=0
$$

Then for $\psi$ pure, in the frame of the Cartan map, with the usual (local) choice $\varphi_{0} / \varphi_{1}=\zeta$, we may express $\chi$ in terms of $\varphi$ and we will have $Z_{\mu}=Z_{\mu}(\zeta)$ and, as before, we may find the coordinates $T_{\mu}(\zeta)$ of the searched null-curve by integration.

Let us now consider explicit coordinates: from (15) we have $Z_{\mu}=\frac{1}{2}\left\langle\varphi \sigma_{\mu} \chi\right\rangle$ and the Cartan-map equation is:

$$
\chi\rangle\left\langle\varphi=\left|\begin{array}{cc}
\chi_{0} \varphi_{1} & -\chi_{0} \varphi_{0} \\
\chi_{1} \varphi_{1} & -\chi_{1} \varphi_{0}
\end{array}\right|=\left|\begin{array}{cc}
Z_{0}+Z_{3} & Z_{1}-i Z_{2} \\
Z_{1}+i Z_{2} & Z_{0}-Z_{3}
\end{array}\right|=Z^{\mu} \sigma_{\mu},\right.
$$

from which we derive the equality of projective coordinates:

$$
\begin{aligned}
& Z_{3}+Z_{0}=\chi_{0} \varphi_{1} \rightarrow \chi_{0}(\zeta)=t_{3}+t_{0} \\
& Z_{3}-Z_{0}=\chi_{1} \varphi_{0} \rightarrow \zeta \chi_{1}(\zeta)=t_{3}-t_{0} \\
& Z_{1}-i Z_{2}=-\chi_{0} \varphi_{0} \rightarrow-\zeta \chi_{0}(\zeta)=t_{1}-i t_{2} \\
& Z_{1}+i Z_{2}=\chi_{1} \varphi_{1} \rightarrow \chi_{1}(\zeta)=t_{1}+i t_{2}
\end{aligned}
$$

where equalities on the right-hand side, with $\chi_{j}(\zeta)$ holomorphic, are obtained after the choice $\zeta=\varphi_{0} / \varphi_{1}$ (for $\varphi_{1} \neq 0$ ) as local projective coordinate representing $\varphi_{t}=\left|\begin{array}{l}\zeta \\ 1\end{array}\right|$. Then the equation $d T^{\mu} d T_{\mu}=0$ may be solved by:

$$
\begin{aligned}
T_{3}+T_{0} & =\int \chi_{0}(\zeta) d \zeta \\
T_{3}-T_{0} & =\zeta \int \chi_{1}(\zeta) d \zeta-\int d \zeta \int \chi_{1}(\zeta) d \zeta \\
T_{1}-i T_{2} & =-\zeta \int \chi_{0}(\zeta) d \zeta+\int d \zeta \int \chi_{0}(\zeta) d \zeta \\
T_{1}+i T_{2} & =\int \chi_{1}(\zeta) d \zeta
\end{aligned}
$$

or, in differential form:

$$
\begin{array}{ll}
T_{0}(\zeta)=f^{\prime}-\zeta g^{\prime}+g, & T_{3}(\zeta)=f^{\prime}+\zeta g^{\prime}-g, \\
i T_{2}(\zeta)=g^{\prime}+\zeta f^{\prime}-f, & T_{1}(\zeta)=g^{\prime}-\zeta f^{\prime}+f
\end{array}
$$

where the arbitrary holomorphic functions $f(\zeta)$, and $g(\zeta)$ are defined by

$$
f(\zeta)=\frac{1}{2} \int d \zeta \int \chi_{0}(\zeta) d \zeta, \quad g(\zeta)=\frac{1}{2} \int d \zeta \int \chi_{1}(\zeta) d \zeta
$$

\footnotetext{
$\overline{{ }^{8} \text { In fact for }} \gamma_{j}, \gamma_{0}=\left|\begin{array}{cc}0 & \sigma_{j},-1 \\ \sigma_{j},+1 & 0\end{array}\right|$ we have: $Z_{1}=\frac{1}{2}\left(\varphi_{0} \chi_{0}-\varphi_{1} \chi_{1}\right), \quad Z_{2}=\frac{i}{2}\left(\varphi_{0} \chi_{0}+\varphi_{1} \chi_{1}\right)$, $Z_{3}=\frac{1}{2}\left(\varphi_{1} \chi_{0}+\varphi_{0} \chi_{1}\right), Z_{0}=\frac{1}{2}\left(\varphi_{1} \chi_{0}-\varphi_{0} \chi_{1}\right)$, and then $Z_{\mu} Z^{\mu}=0$. Geometrically $Z_{\mu}$ is the null intersection of the two planes Cartan-equivalent to $\varphi$ and $\chi$
} 
Equation (18) has been also obtained by Eisenhart [13] and by Shaw [14] from twistor theory ${ }^{9}$.

By general theorems $[10,15]$ then:

$$
X_{\mu}(u, v)=\operatorname{Re} T_{\mu}(\zeta)
$$

are the most general equations ${ }^{10}$ for a minimal surface in $R^{3,1}$; (and for $\operatorname{Re} T_{i}$, $\operatorname{Im} T_{0}$ the same in $\left.\mathbb{R}^{4}\right) . X_{\mu}(u, v)$ satisfy the equations defining minimal surfaces in $\mathbb{R}^{3,1}$,

$$
\frac{\partial^{2} X_{\mu}}{\partial u^{2}}+\frac{\partial^{2} X_{\mu}}{\partial v^{2}}=0 ; \quad\left(\frac{\partial X_{\mu}}{\partial u}+i \frac{\partial X_{\mu}}{\partial v}\right)^{2}=0 .
$$

In order to find the corresponding string equations the simplest thing to do will be to consider $\mathbb{R}^{3,2}$ spinors which may be real since $\mathrm{Cl}(3,2)=R(4) \oplus R(4)$. And in fact we may repeat the same procedure as for $\mathbb{R}^{4,1}$ only now $\psi=\left|\begin{array}{l}\phi_{+} \\ \phi_{-}\end{array}\right| ; \gamma_{j}=\left|\begin{array}{ll}0 & \varepsilon_{j} \\ \varepsilon_{j} & 0\end{array}\right|$; $\gamma_{0}=\left|\begin{array}{rr}0 & -1 \\ 1 & 0\end{array}\right|$ and $\varepsilon_{j}$ and $\phi_{+}, \phi_{-}$are the same as those of the preceding paragraph [see Eq. (9) and following].

The Cartan-map Eq. (14) is now real and complex $Z_{\mu}$ are substituted by real $K_{\mu}=\frac{1}{4}\left\langle\psi \gamma_{\mu} \gamma_{5} \psi\right\rangle$ and we easily obtain instead of (17):

$$
\begin{gathered}
K_{3}+K_{0}=\phi_{0}^{-} \phi_{1}^{+}, \quad K_{3}-K_{0}=\phi_{1}^{-} \phi_{0}^{+}, \\
K_{1}-K_{2}=-\phi_{0}^{-} \phi_{0}^{+}, \quad K_{1}+K_{2}=\phi_{1}^{-} \phi_{1}^{+},
\end{gathered}
$$

satisfying $K_{1}^{2}-K_{2}^{2}+K_{3}^{2}-K_{0}^{2}=0$.

We have now two possibilities of local projective coordinates: either $\phi_{0}^{+} / \phi_{1}^{+}$ $=\alpha_{+}=\sigma+\tau$ or $\phi_{0}^{-} / \phi_{1}^{-}=\alpha_{-}=\sigma-\tau$; correspondingly we may interpret $K_{\mu}$ as null tangents $t_{\mu}^{+}$and $t_{\mu}^{-}$to two curves in $\mathbb{R}^{2,2}$ parametrized by $\alpha_{+}$and $\alpha_{-}$:

$$
\begin{aligned}
& t_{3}^{+}+t_{0}^{+}=\phi_{0}^{-}\left(\alpha_{+}\right), \quad t_{3}^{-}+t_{0}^{-}=\phi_{1}^{+}\left(\alpha_{-}\right), \\
& t_{3}^{+}-t_{0}^{+}=\phi_{1}^{-}\left(\alpha_{+}\right) \alpha_{-}, \quad t_{3}^{-}-t_{0}^{-}=\phi_{1}^{+}\left(\alpha_{-}\right) \alpha_{+}, \\
& t_{1}^{+}-t_{2}^{+}=-\phi_{0}^{-}\left(\alpha_{+}\right) \alpha_{+}, \quad t_{1}^{-}-t_{2}^{-}=-\phi_{0}^{+}\left(\alpha_{-}\right), \\
& t_{1}^{+}+t_{2}^{+}=\phi_{1}\left(\alpha_{+}\right), \quad t_{1}^{-}+t_{2}^{-}=\phi_{1}^{+}\left(\alpha_{-}\right) \alpha_{-} .
\end{aligned}
$$

The two $\chi_{\mu}^{+}, \chi_{\mu}^{-}$curves are obtained by integration $\chi_{\mu}^{ \pm}\left(\alpha^{ \pm}\right)=\int t_{\mu}^{ \pm} d \alpha^{ \pm}$, and the string in $\mathbb{R}^{2,2}$ is then

$$
\chi_{\mu}(\sigma, \tau)=\chi_{\mu}(0,0)+\int_{0}^{\sigma+\tau} t_{\mu}^{+}\left(\alpha^{+}\right) d \alpha^{+}+\int_{0}^{\sigma-\tau} t_{\mu}^{-}\left(\alpha^{-}\right) d \alpha^{-},
$$

\footnotetext{
${ }^{9}$ We could also have taken the two complex Weyl spinors $\varphi_{1}$ and $\chi_{2}$ as belonging to different Dirac spinors $\psi_{1}$ and $\psi_{2}$ respectively. In this case $\psi$ would have represented a $\mathbb{R}^{4,2}$ pure spinor (semi spinor in Cartan terminology [3]) or twistor. The Cartan-map for $\mathbb{R}^{4,2}$ will include both real spinors and twistors. However these might be less adapted for the generation of strings, since Weyl $\mathbb{R}^{3,1}$-spinors are always complex $\left(\mathrm{Cl}^{+}(3,1)=\mathbb{C}(2) \oplus \mathbb{C}(2)\right)$. An interesting exceptional case may be $\mathbb{R}^{9,1}$ since $\mathrm{Cl}(9,1)=\mathrm{Cl}(1,1) \otimes \mathrm{Cl}(8,0)$ and for the periodicity theorem $\mathbb{R}^{9,1}$ semispinors (or Weyl) are real and then might originate strings

${ }^{10}$ It is interesting to note that solution $\left(18^{\prime}\right)$ of $d T^{\mu} d T_{\mu}=0$ has been incidentally found and extensively studied in the context of null vectors and isotropic planes in $\mathbb{C}^{4}$ and $\mathbb{R}^{3,1}$ by Eisenhart in 1911 [13] before the discovery of spinors
} 
which can be explicitly expressed from (20). It is easy to verify that $\chi_{\mu}(\sigma, \tau)$ are solutions of the string equations in $\mathbb{R}^{2,2}$.

\section{Strings in $\mathbb{R}^{3,1}$-Minkowski Space Generated by Majorana Space-time Spinors}

Since $\mathrm{Cl}(2,2)=\mathrm{Cl}(3,1)=\mathbb{R}(4)$, it must be possible by this procedure to generate also $\mathbb{R}^{3,1}$ space-time strings. To this end it will be necessary to start from a Majorana space-time spinor. We may obtain it taking as generating $\mathrm{Cl}(3,1)$ real matrices:

$$
\begin{gathered}
\gamma_{1}=\sigma_{3} \otimes \sigma_{1} ; \quad \gamma_{2}=\sigma_{3} \otimes \sigma_{3} ; \quad \gamma_{3}=\sigma_{1} \otimes \mathbb{1} ; \quad \gamma_{0}=\varepsilon_{2} \otimes \mathbb{1} \\
\tilde{\gamma}_{5}=\gamma_{1} \gamma_{2} \gamma_{3} \gamma_{0}=\sigma_{3} \otimes \varepsilon_{2}, \quad\left(\tilde{\gamma}_{5}^{2}=-1\right)
\end{gathered}
$$

then since $\sigma_{1}, \sigma_{3}$ generate $\mathrm{Cl}(2,0)$ the Majorana spinor $\psi$ will have the form:

$$
\psi=\left|\begin{array}{l}
u \\
v
\end{array}\right|
$$

where $u$ and $v$ are real $\mathbb{R}^{2,0}$ spinors (non-pure: they are real $\mathbb{R}^{2,1}$-pure spinors where the extra time-generator $\varepsilon_{2}$ is contained in $\tilde{\gamma}_{5}$ ) or Majorana spinors for 3-dimensional space-time.

From the Cartan-map ${ }^{11}$ we obtain now the equation $\left(\left\langle\psi:=\psi^{T} \gamma_{1} \gamma_{2} \gamma_{3}\right)\right.$ :

$$
\psi\rangle\left\langle\psi=\frac{1}{2} f^{\mu v}\left[\gamma_{\mu}, \gamma_{v}\right]+\frac{1}{2} k_{\mu} \gamma^{\mu} \tilde{\gamma}_{5},\right.
$$

and the real, lightlike (or null or optical) vector $k_{\mu}=\frac{1}{2}\left\langle\psi \gamma_{\mu} \tilde{\gamma}_{5} \psi\right\rangle$ may be expressed in terms of the real Majorana spinor components (we introduced the factor $1 / 2$ for sake of economy):

$$
\begin{aligned}
k_{3}+k_{0} & =-\left(u_{0}^{2}+u_{1}^{2}\right), & & k_{3}-k_{0}=v_{0}^{2}+v_{1}^{2}, \\
k_{1} & =u_{1} v_{0}+u_{0} v_{1}, & & k_{2}=u_{0} v_{0}-u_{1} v_{1},
\end{aligned}
$$

and it is easily verified that $k_{\mu} k^{\mu}=0$ for any $u$ and $v$.

We may now think of $k_{\mu}$ as optical tangents to two optical curves in $\mathbb{R}^{3,1}$ and by integration we could obtain a string in terms of them. We wish to show that all this may naturally be obtained by selecting appropriate local projective charts to represent the Cartan-map above. We easily obtain in fact:

$$
u\rangle\left\langle=\left|\begin{array}{cc}
u_{0} u_{1} & -u_{0}^{2} \\
u_{1}^{2} & -u_{0} u_{1}
\end{array}\right|=\left|\begin{array}{cc}
f_{2}^{+} & f_{1}^{+}-k^{+} \\
f_{1}^{+}+k^{+} & -f_{2}^{+}
\end{array}\right|,\right.
$$

where

$$
f_{j}^{+}-f_{j 0}+f_{j 3} ; \quad k^{+}=\frac{1}{2}\left(k_{0}+k_{3}\right) .
$$

From these, going to projective local coordinates $\frac{u_{0}}{u_{1}}=f_{20}+f_{23}=\alpha_{+}=\sigma+\tau$, we obtain the optical vector $t_{\mu}\left(t_{\mu} t^{\mu}=0\right)$ tangent to the optical curve in $\mathbb{R}^{3,1}$ :

$$
\begin{array}{cc}
t_{3}^{+}+t_{0}^{+}=-\left(1+\alpha_{+}^{2}\right), & t_{3}^{+}-t_{0}^{+}=v_{0}^{2}\left(\alpha_{+}\right)+v_{1}^{2}\left(\alpha_{+}\right), \\
t_{1}^{+}=v_{0}\left(\alpha_{+}\right)+\alpha_{+} v_{1}\left(\alpha_{+}\right), & t_{2}^{+}=\alpha_{+} v_{0}\left(\alpha_{+}\right)-v_{1}\left(\alpha_{+}\right) .
\end{array}
$$

${ }^{11}$ Since $\tilde{\gamma}_{5}^{2}=-1, \psi$ now represents a real, pure, $R^{3,2}$-spinor 
From

$$
v\rangle\left\langle v=\left|\begin{array}{cc}
v_{0} v_{1} & -v_{0}^{2} \\
v_{1}^{2} & -v_{0} v_{1}
\end{array}\right|=\left|\begin{array}{cc}
-f_{2}^{-} & -f_{1}^{-}-k^{-} \\
-f_{1}^{-}+k^{-} & f_{2}^{-}
\end{array}\right|,\right.
$$

where $f_{j}^{-}=f_{j 3}-f_{j 0} ; k^{-}=\frac{1}{2}\left(k_{3}-k_{0}\right)$. Choosing the corresponding local projective coordinates: $-\frac{v_{1}}{v_{0}}=f_{23}-f_{20}=\alpha_{-}=\sigma-\tau$ we obtain the optical tangents of another line in $\mathbb{R}^{3,1}$ :

$$
\begin{aligned}
t_{3}^{-}+t_{0}^{-} & =-\left[u_{0}^{2}\left(\alpha_{-}\right)+u_{1}^{2}\left(\alpha_{-}\right)\right], \quad t_{3}^{-}-t_{0}^{-}=1+\alpha_{-}^{2}, \\
t_{1}^{-} & =u_{1}\left(\alpha_{-}\right)-\alpha_{-} u_{0}\left(\alpha_{-}\right), \quad t_{2}^{-}=u_{0}\left(\alpha_{-}\right)+\alpha_{-} u_{1}\left(\alpha_{-}\right) .
\end{aligned}
$$

From these it is easy to give the equation of a string in space-time expressed in terms of the two optical lines naturally generated in the projective space of a Majorana space-time spinor:

$$
\chi_{\mu}(\sigma, \tau)=\chi_{\mu}(0,0)+\int_{0}^{\sigma+\tau} t_{\mu}^{+}\left(\alpha_{+}\right) d \alpha_{+}+\int_{0}^{\sigma-\tau} t_{\mu}^{-}\left(\alpha_{-}\right) d \alpha_{-},
$$

where

$$
t_{\mu}^{ \pm}\left(\alpha_{ \pm}\right)=\frac{1}{2}\left\langle\psi\left(\alpha_{ \pm}\right) \gamma_{\mu} \tilde{\gamma}_{5} \psi\left(\alpha_{ \pm}\right)\right\rangle
$$

and

$$
\left.\left.\psi\left(\alpha_{-}\right)\right\rangle:=\left|\begin{array}{c}
u_{0}\left(\alpha_{-}\right) \\
u_{1}\left(\alpha_{-}\right) \\
v_{0}=1 \\
v_{1}=-\alpha_{-}
\end{array}\right| ; \quad \psi\left(\alpha_{+}\right)\right\rangle:=\left|\begin{array}{c}
u_{0}=\alpha_{+} \\
u_{1}=1 \\
v_{0}\left(\alpha_{+}\right) \\
v_{1}\left(\alpha_{+}\right)
\end{array}\right|
$$

It is easily verified that $\chi_{\mu}(\sigma, \tau)$ satisfy the string equations in $\mathbb{R}^{3,1}$ :

$$
\frac{\partial^{2} \chi_{\mu}}{\partial \sigma^{2}}-\frac{\partial^{2} \chi_{\mu}}{\partial \tau^{2}}=0, \quad \frac{\partial \chi_{\mu}}{\partial \sigma} \frac{\partial \chi^{\mu}}{\partial \sigma}+\frac{\partial \chi_{\mu}}{\partial \tau} \frac{\partial \chi^{\mu}}{\partial \tau}=0, \quad \frac{\partial \chi_{\mu}}{\partial \sigma} \frac{\partial \chi^{\mu}}{\partial \tau}=0
$$

\section{Conclusion and Outlook}

This simple application of the Cartan-map which generates minimal surfaces from complex spinors and strings from real ones could be generalized through the use of other charts and atlas and extended to higher dimensional spaces where, coherently with the spinor construction, coordinates in projective spaces will be obtained, containing locally ordinary space-time; furthermore, pureness constraints will play interesting roles.

The next obvious step is to add to the selected charts the excluded sets $\left(\varphi_{1}=0\right.$, $\left.\chi_{0}=0 \ldots\right)$ in order to put in evidence the global and topological properties. This point will be essential for the discussion of closed strings, in fact it is clear that open, infinite strings in the local charts may instead appear closed when the point at infinity corresponding, say to $\varphi_{1}=0,(\zeta=\infty)$, is added to the chart. These compactifications may become important, and contribute to the elimination of divergences (both infrared and ultraviolet), when the method is applied to 
conformal extensions of space-time [5], since then the compactifying sets lie on the light cone at infinity. These and other matters such as the generation of similar results from the Cartan-map identification of spinor directions with coset space elements in projective spaces and the role of the stability groups for the generation of string structures; the embedding of spinors in Clifford algebras (minimal ideals) with consequent non-commutative properties, besides obvious dynamical aspects, will be the subject of further work.

At the present stage we wish to stress that the possible common origin of minimal surfaces and strings from complex and real null-vectors (optical-vectors in space-time) and, after Cartan-map, from complex and real spinors might furnish a firm geometrical basis to the conjecture that strings are fundamental objects for the representation of elementary natural phenomena in a similar way as minimal surfaces have been and are, and this may be simply due to the close connection of strings and minimal surfaces with real and complex spinors respectively, which makes them the natural intermediaries between the fundamental, but difficult to imagine, null-vector geometry and the easily visualizable, but not elementary, euclidean one.

Acknowledgements. The author wishes to express his gratitude to Prof. Tullio Regge and Dr. L.P. Hughston for bringing to his attention the old papers of K. Weierstrass and of L. P. Eisenhart, respectively, and to Prof. Paolo Furlan for helpful discussions.

\section{References}

1. Enneper, A.: Z. Math. Phys. 9, 96 (1864);

Weierstrass, K.: Monatsberichte der Berliner Akademie 612 (1866)

2. Cartan, E.: Bull. Soc. Math. Fr. 41, 53 (1913)

3. Cartan, E.: La théorie des spineures. Paris: Hermann (1938)

4. Budinich, P., Trautman, A.: Remarks on pure spinors. Lett. Math. Phys. 11, 315 (1986)

5. Budinich, P.: Pure spinors for conformal extensions of space-time. I.S.A.S. Internal report 1/E.P./86

6. Fermi, E.: Nuovo Cim. 11, 1 (1934)

Heisenberg, W.: Zur Theorie der „Schauer“ in der Höhenstrahlung. Z. Phys. 101, 533 (1936); Quantum theory of fields and elementary particles. Rev. Mod. Phys. 29, 269 (1957)

Gell-Mann, M.: Symmetries of baryons and mesons. Phys. Rev. 125, 1067 (1962)

7. Penrose, R., Rindler, W.: Spinors and space-time. Cambridge: Cambridge University Press 1984 and 1986;

Budinich, P.: Czech. J. Phys. B 29, 6 (1979);

Furlan, P., Raczka, R.: Nonlinear spinor representations. I.S.A.S. Preprint;

Benn, I.M., Tucker, R.W.: Pure spinors and real Clifford algebras. University of Lancaster Preprint (1984);

Dabrowski, L., Trautman, A.: Spinor structures on spheres and projective spaces. J. Math. Phys. (to appear);

Hughston, L.P., Hurd, T.R.: A $C P^{5}$ calculus for space-time fields. Phys. Rep. 100, 273 (1983); Caianiello, E.R., Giovannini, A.: Pure spinors as Pfaffians connecting Clifford and Grassmann algebras. Lett. Nuovo Cimento 34, 301 (1982);

Flato, M., Fronsdal, C.: Quarks or singletons. U.C.L.A. Preprint (1985)

8. Veneziano, G.: Construction of a crossing symmetric, Regge-behaved amplitude for linearly rising trajectories. Nuovo Cimento 57 A, 190 (1968);

Fubini, S., Veneziano, G.: Level structure of dual-resonance models. Nuovo Cimento 64A, 811 (1969) 
Nambu, Y.: Proc. Int. Conf. on Symmetries and Quark Models. Wayne State University (1969);

Nielsen, H.: XV Int. Conf. on High Energy Physics, Kiev (1970);

Susskind, L.: Dual-symmetric theory of hadrons. I. Nuovo Cimento 69A, 457 (1970)

9. Weinberg, S.: Invited paper at the Niels Bohr Centennial, Kopenhagen (1985);

Witten, E.: Non commutative geometry and string field theory. Princeton-Preprint (October 1985) (to be consulted for further references)

10. Nitsche, J.C.C.: Vorlesungen über Minimalfläche. Berlin, Heidelberg, New York: Springer 1975

11. Hitchin, N.J.: Monopoles and geodesics. Commun. Math. Phys. 83, 579 (1982)

12. Trautman, A.: Optical Structures in Relativistic Theories: Asterisque, hors série 401 (1985)

13. Eisenhart, L.P.: Am. J. Math. 34, 216 (1912)

14. Shaw, W.T.: Class Quantum Grav. 2, 113 (1985)

15. Lawson, H.B.: Lectures on minimal submanifolds. Berkeley: Publish or Perish (1980)

Communicated by G. Parisi

Received February 4, 1986; in revised form July 7, 1986

Note added in proofs: $\psi=\left|\begin{array}{c}\varphi \\ \dot{\varphi}\end{array}\right|$ represents an $\mathbb{R}^{3,1}$ Majorana spinor. 
